\title{
Enumeration of orientable coverings of a non-orientable manifold
}

\author{
Jin Ho Kwak ${ }^{1 \dagger}$ and Alexander Mednykh ${ }^{2 \ddagger}$ and Roman Nedela ${ }^{3 \S}$ \\ ${ }^{1}$ Pohang University of Science and Technology, Pohang, 790-784 Korea \\ ${ }^{2}$ Institute of Mathematics, Novosibirsk State University, 630090, Novosibirsk, Russia, \\ Universidad Técnica Federico Santa María, Avenida España 1680, Casilla 110-V, Valparaso. Chile \\ ${ }^{3}$ Institute of Mathematics, Slovak Academy of Science, Severná 5, 97549 Banská Bystrica, Slovakia
}

\begin{abstract}
In this paper we solve the known V.A. Liskovets problem (1996) on the enumeration of orientable coverings over a non-orientable manifold with an arbitrary finitely generated fundamental group. As an application we obtain general formulas for the number of chiral and reflexible coverings over the manifold. As a further application, we count the chiral and reflexible maps and hypermaps on a closed orientable surface by the number of edges. Also, by this method the number of self-dual and Petri-dual maps can be determined. This will be done in forthcoming papers by authors.
\end{abstract}

Keywords: non-orientable manifold, fundamental group, conjugacy classes of subgroups, surface covering, enumeration, chiral pairs

\section{Introduction}

Let $\mathcal{M}$ be a connected non-orientable manifold. Denote by $\Gamma=\pi_{1}(\mathcal{M})$ the fundamental group and by $\tilde{\mathcal{M}}$ the universal covering of $\mathcal{M}$. Identify $\Gamma$ with the group of covering transformations of $\tilde{\mathcal{M}} \rightarrow \mathcal{M}$. We note that $\tilde{\mathcal{M}}$ is always orientable and $\Gamma$ acts on $\tilde{\mathcal{M}}$ as a group of homeomorphisms.

Denote by $\Gamma^{+}$a subgroup of index two in $\Gamma$ consisting of all orientation preserving homeomorphisms. Then $\mathcal{M}^{+}=\tilde{\mathcal{M}} / \Gamma^{+}$is an orientable double of $\mathcal{M}$ with fundamental group $\Gamma^{+}=\pi_{1}\left(\mathcal{M}^{+}\right)$. The following facts from algebraic topology are well known (5).

Let $\pi: \mathcal{U} \rightarrow \mathcal{M}$ be an $n$-fold covering of $\mathcal{M}$. Then the fundamental group $K=\pi_{1}(\mathcal{U})$ is contained as a subgroup of index $n$ in the group $\Gamma=\pi_{1}(\mathcal{M})$. Conversely, any subgroup of index $n$ in $\Gamma$ is the fundamental group of an $n$-fold covering of $\mathcal{M}$. Moreover, if $\mathcal{U}^{+}$is orientable then the number $n=2 \mathrm{~m}$ is even and the group $K$ is contained as a subgroup of index $m$ in the group $\Gamma^{+}$. In this case, $\mathcal{U}^{+}$is an $m$-fold covering of the manifold $\mathcal{M}^{+}$.

\footnotetext{
${ }^{\dagger}$ Supported by the Korea Research Foundation Grant funded Korean Government (KRF-2007-313-C00011).

${ }^{\ddagger}$ Supported by the RFBR (grant 06-01-00153) and Fondecyt (grants 7050189, 1060378).

$\S$ Supported by Slovak Academy of Science (VEGA) and by Science and Technology Assistance Agency under the contract No. APVV-51-012502.
} 
Two coverings $\pi: \mathcal{U} \rightarrow \mathcal{M}$ and $\pi^{\prime}: \mathcal{U}^{\prime} \rightarrow \mathcal{M}$ are equivalent (or isomorphic) if there exists a homeomorphism $h: \mathcal{U} \rightarrow \mathcal{U}^{\prime}$ such that $\pi=\pi^{\prime} \circ h$. An orientable covering $\pi: \mathcal{U}^{+} \rightarrow \mathcal{M}$ is called reflexible if there exists an orientation reversing homeomorphism $h: \mathcal{U}^{+} \rightarrow \mathcal{U}^{+}$such that $\pi \circ h=\pi$ and irreflexible (or chiral) otherwise. Irreflexible coverings are divided into chiral pairs of twins. Two twins are non-equivalent as coverings over $\mathcal{M}^{+}$, but have to be equivalent as coverings over $\mathcal{M}$.

Coverings $\pi: \mathcal{U} \rightarrow \mathcal{M}$ and $\pi^{\prime}: \mathcal{U}^{\prime} \rightarrow \mathcal{M}$ are equivalent if and only if the corresponding subgroups $\pi_{1}(\mathcal{U})$ and $\pi_{1}\left(\mathcal{U}^{\prime}\right)$ are conjugate in $\Gamma$. Hence, the number of non-equivalent $n$-fold coverings of $\mathcal{M}$ coincides with the number $c_{\Gamma}(n)$ of conjugacy classes of subgroups of index $n$ in the group $\Gamma$.

Recall that the fundamental group of a bordered surface $\mathcal{S}$ is a free group $\Gamma=F_{r}$ of $\operatorname{rank} r=1-\chi(\mathcal{S})$, where $\chi(\mathcal{S})$ is the Euler characteristic of $\mathcal{S}$. It this case the number $c_{\Gamma}(n)$ was determined by V. Liskovets ((15), (16)), J. H. Kwak and J. Lee (12), and M. Hofmeister (6). For the fundamental group $\Gamma$ of closed orientable and non-orientable surfaces the number $c_{\Gamma}(n)$ was given by A. Mednykh (19) and A. Mednykh and G. Pozdnyakova (23), respectively.

During the discussion at Dresden University between V. A. Liskovets and one of the authors in 1996 the following problem was stated:

Liskovets problem. Find the number of non-equivalent n-fold orientable coverings of a given nonorientable manifold with a finitely generated fundamental group.

The main purpose of the paper is to give a solution of the Liskovets problem (Theorem 3). As an application, we enumerate reflexible coverings and of chiral pairs of coverings over a non-orientable manifold with a finitely generated fundamental group. These results form a background for counting chiral pairs of maps and hypermaps on closed orientable surface. It will be done in a forthcoming paper by A. Breda, R. Nedela and A. Mednykh (1). The general formula for the number of reflexible coverings (Theorem 8) allows also to count self-dual and Petri-dual maps and other combinatorial objects.

\section{Preliminaries}

Following J. Šráň and M. S̆koviera (24) we define a group with sign structure to be a pair $(\Gamma, \omega)$, where $\Gamma$ is a group and $\omega: \Gamma \rightarrow \mathbb{Z}_{2}=\{-1,1\}$ is a homomorphism. Each sign structure (or orientation) $\omega$ is uniquely determined by a subgroup $H=\operatorname{Ker}(\omega)$ of index at most two in $\Gamma$. Equivalently, one can define a group with sign structure as a pair $(\Gamma, H)$, where $H$ is a subgroup of index one or two in $\Gamma$.

Let $(\Gamma, \omega)$ be a group with sign structure. The image of an element $g \in \Gamma$ under homomorphism $\omega$ will be referred as the sign of $g$. Elements with sign +1 will be called positive and those with sign -1 will be called negative. The set of positive elements of $\Gamma$ forms a subgroup, denoted by $\Gamma^{+}$, of index one or two in $\Gamma$. The set of negative elements $\Gamma^{-}$is a coset of $\Gamma^{+}$in $\Gamma$ or empty. In the latter case the corresponding sign structure will be called trivial.

A subgroup $K<\Gamma$ is called to be orientable (with respect to $\omega$ ) if $K \subset \operatorname{Ker}(\omega)$ and non-orientable otherwise. For any orientable and non-orientable subgroup $K<\Gamma$ the induced sign structure $\left.\omega\right|_{K}: K \rightarrow$ $\mathbb{Z}_{2}$ is trivial or non-trivial, respectively. The given definitions are justified by the following topological observations.

Let $\mathcal{M}$ be a manifold with the fundamental group $\Gamma=\pi_{1}(\mathcal{M})$. Then $\Gamma$ acts as a homeomorphism group on the universal covering $\tilde{\mathcal{M}}$ of the manifold $\mathcal{M}$. Denote by $\Gamma^{+}$the group of all orientation preserving 
homeomorphisms of $\Gamma$. The pair $\left(\Gamma, \Gamma^{+}\right)$uniquely defines a sign structure $\omega: \Gamma \rightarrow \mathbb{Z}_{2}$ with $\operatorname{Ker}(\omega)=$ $\Gamma^{+}$.

We note that the cyclic group $\Gamma=\mathbb{Z}_{2}$ is the fundamental group of a non-orientable projective plane $\mathcal{P}^{2}$ and of the orientable projective three-space $\mathcal{P}^{3}$. In the first case, $\Gamma^{+}=\langle 1\rangle$ and $(\Gamma, \omega)$ is a non-orientable group. In the second, $\Gamma^{+}=\mathbb{Z}_{2}$ and $(\Gamma, \omega)$ is an orientable group.

EXAMPLE 1 Let $F_{r}=\left\langle x_{1}, x_{2}, \ldots, x_{r}\right\rangle$ be a free group of rank $r$ and $\omega: F_{r} \rightarrow \mathbb{Z}_{2}=\{-1,1\}$ is the epimorphism defined by $\omega\left(x_{i}\right)=(-1)^{p_{i}}, i=1,2, \ldots, r$, where at least one of the integers $p_{1}, p_{2}, \ldots, p_{r}$ is odd. Then $\left(F_{r}, \omega\right)$ be the group with sign structure.

EXAMPLE 2 Let $\Lambda_{p}=\left\langle x_{1}, x_{2}, \ldots, x_{p}: x_{1}^{2} x_{2}^{2} \cdots x_{p}^{2}=1\right\rangle$ be the fundamental group of a closed nonorientable surface of genus $p$ and $\omega: \Lambda_{p} \rightarrow \mathbb{Z}_{2}=\{1,-1\}$ be the epimorphism defined by $\omega\left(x_{i}\right)=$ $(-1)^{q_{i}}, i=1, \ldots, p$, where at least one of the integers $q_{1}, q_{2}, \ldots, q_{p}$ is odd. Then $\left(\Lambda_{p}, \omega\right)$ is a group with sign structure.

EXAMPLE 3 Let $\mathbb{Z}_{\ell}=\left\langle x: x^{\ell}=1\right\rangle$ be a finite cyclic group of order $\ell$. If $\ell$ is odd then there is only one (trivial) orientation on the group $\mathbb{Z}_{\ell}$ given by $\omega(x)=1$. If $\ell$ is even there is only one non-trivial orientation defined by $\omega(x)=-1$. In both cases we will say that $\omega$ is the canonical orientation of $\mathbb{Z}_{\ell}$.

Let $\Gamma=(\Gamma, \omega)$ and $A=(A, \eta)$ be two groups with sign structure. A homomorphism $\psi: \Gamma \rightarrow A$ is said to be orientation preserving if $\psi\left(\Gamma^{+}\right) \subseteq A^{+}$and $\psi\left(\Gamma^{-}\right) \subseteq A^{-}$. An epimorphism $\psi: \Gamma \rightarrow$ $A$ is orientation preserving if and only if its kernel is a subgroup of $\Gamma^{+}$. Note that the derived group $\Gamma^{\prime}=[\Gamma, \Gamma]<\Gamma^{+}$. Denote by $\operatorname{Hom}^{+}(\Gamma, A), \mathrm{Epi}^{+}(G, A)$ the respective sets of orientation preserving homomorphisms and epimorphisms $\Gamma \rightarrow A$. We consider $H_{1}(\Gamma)=\Gamma / \Gamma^{\prime}$ as a group with sign structure whose positive elements are $\Gamma^{+} / \Gamma^{\prime}$.

Thus we have the following auxiliary result.

Lemma 1 Let $\Gamma=(\Gamma, \omega)$ and $A=(A, \eta)$ be groups with sign structure and let $A$ be abelian. Then $\left|\mathrm{Hom}^{+}(\Gamma, A)\right|=\left|\mathrm{Hom}^{+}\left(H_{1}(\Gamma), A\right)\right|$.

Let $\Gamma$ and $\mathcal{A}$ be two finitely generated groups. Denote by $\operatorname{Hom}(\Gamma, \mathcal{K})(\operatorname{resp} . \operatorname{Epi}(\Gamma, \mathcal{K})$ ) the sets of homomorphisms (respectively, epimorphisms) from $\Gamma$ to $\mathcal{K}$. By the Philip Hall inversion formula (3) we obtain

$$
|\operatorname{Epi}(\Gamma, \mathcal{A})|=\sum_{\mathcal{K} \leq \mathcal{A}} \mu(\mathcal{K})|\operatorname{Hom}(\Gamma, \mathcal{K})|,
$$

where $\mu$ is the Möbius function for the group $\mathcal{A}$ which assigns an integer $\mu(\mathcal{K})$ to each subgroup $\mathcal{K}$ of $\mathcal{A}$ by the recursive formula

$$
\sum_{H \geq K} \mu(H)=\delta_{K, \mathcal{A}}= \begin{cases}1 & \text { if } K=\mathcal{A} \\ 0 & \text { if } K<\mathcal{A} .\end{cases}
$$

Let $\Gamma=(\Gamma, \omega)$ and $\mathcal{A}=(\mathcal{A}, \eta)$ be groups with non-trivial sign structure. That is the groups $\Gamma^{+}=$ $\operatorname{Ker} \omega$ and $\mathcal{A}^{+}=\operatorname{Ker} \eta$ are subgroups of index two in the groups $\Gamma^{+}$and $\mathcal{A}^{+}$, respectively. We set also $\mathcal{K}^{+}=\mathcal{K} \cap \Gamma^{+}$. 
The following generalization of the P. Hall formula has been obtained in (13).

\section{Proposition 1}

$$
\left|\operatorname{Epi}^{+}(\Gamma, \mathcal{A})\right|=\sum_{\substack{\mathcal{K} \leq \mathcal{A} \\ \mathcal{K} \neq \mathcal{K}^{+}}} \mu(\mathcal{K})\left|\operatorname{Hom}^{+}(\Gamma, \mathcal{K})\right| .
$$

\section{Counting conjugacy classes of subgroups}

Consider a finitely generated group $\Gamma$. Let $\mathcal{P}$ be a property of subgroups of $\Gamma$, which is invariant under conjugation (for instance: to be normal, to be torsion free, to be orientable and so on). By a slight modification of arguments from the paper (21) we get the following result obtained earlier in (22)

Theorem 1 Let $\Gamma$ be a finitely generated group. Then the number of conjugacy classes of subgroups of index $n$ in the group $\Gamma$ having property $\mathcal{P}$ is given by the formula

$$
c_{\Gamma}^{\mathcal{P}}(n)=\frac{1}{n} \sum_{\substack{\ell \mid n \\ \ell m=n}} \sum_{K<_{m} \Gamma}\left|\operatorname{Epi}^{\mathcal{P}}\left(K, \mathbb{Z}_{\ell}\right)\right|,
$$

where the sum $\sum_{K<_{m} \Gamma}$ is taken over all subgroups $K$ of index $m$ in the group $\Gamma$ and $\mathrm{Epi}^{\mathcal{P}}\left(K, \mathbb{Z}_{\ell}\right)$ is the set of epimorphisms of the group $K$ onto the cyclic group $\mathbb{Z}_{\ell}$ whose kernel has the property $\mathcal{P}$.

From now on we suppose that $\Gamma=(\Gamma, \omega)$ is a finitely generated group with sign structure. The property $\mathcal{P}=\mathcal{P}^{+}$or $\mathcal{P}=\mathcal{P}^{-}$for the subgroups of $\Gamma$ is "to be orientable" or "to be non-orientable", respectively. Applying Theorem 1 for $\mathcal{P}=\mathcal{P}^{-}$we have the following result:

Theorem 2 Let $\Gamma$ be a finitely generated group with non-trivial sign structure. Then the number of conjugacy classes of non-orientable subgroups of index $n$ in the group $\Gamma$ is given by the formula

$$
c_{\Gamma}^{-}(n)=\frac{1}{n} \sum_{\substack{\ell \mid n \\ \ell m=n}} \sum_{K^{-}<_{m} \Gamma}\left|\mathrm{Epi}^{-}\left(K^{-}, \mathbb{Z}_{\ell}\right)\right|,
$$

where the sum $\sum_{K^{-}<_{m} \Gamma}$ is taken over all non-orientable subgroups of index $m$ in the group $\Gamma$ and $\operatorname{Epi}^{-}\left(K, \mathbb{Z}_{\ell}\right)$ is the set of epimorphisms of the group $K$ onto a cyclic group $\mathbb{Z}_{\ell}$ of order $\ell$ with nonorientable kernel.

Let $\mathcal{M}$ be a connected non-orientable manifold with a finitely generated group $\Gamma$. The group $\Gamma$ acts by homeomorphisms on the universal covering $\tilde{\mathcal{M}}$ of the manifold $\mathcal{M}$. Denote by $\Gamma^{+}$the subgroup of index two in $\Gamma$ consisting of all orientation preserving homeomorphisms. Then $\Gamma$ admits a non-trivial sign structure with the set of positive elements $\Gamma^{+}$. We identify the equivalency classes of orientable coverings of $\mathcal{M}$ with conjugacy classes of orientable subgroups in $\Gamma$. The following theorem gives a general solution of V. A. Liskovets problem 
Theorem 3 Let $\Gamma$ be a finitely generated group with non-trivial sign structure. Denote by $\Gamma^{+}$the group of positive elements of $\Gamma$. Then the number of conjugacy classes of orientable subgroups of index $2 n$ in the group $\Gamma$ is given by the formula

$$
c_{\Gamma}^{+}(2 n)=\frac{1}{2 n} \sum_{\substack{\ell \mid n \\ \ell m=n}}\left(\sum_{K^{+}<_{m} \Gamma^{+}}\left|\operatorname{Epi}\left(K^{+}, \mathbb{Z}_{\ell}\right)\right|+\sum_{K^{-}<_{m} \Gamma}\left|\operatorname{Epi}^{+}\left(K^{-}, \mathbb{Z}_{2 \ell}\right)\right|\right),
$$

where the sum $\sum_{K^{-}<_{m} \Gamma}$ is taken over all non-orientable subgroups of index $m$ in the group $\Gamma$, and $\mathrm{Epi}^{+}\left(K^{-}, \mathbb{Z}_{2 \ell}\right)$ is the set of epimorphisms of the group $K^{-}$onto a cyclic group $\mathbb{Z}_{2 \ell}$ with orientable kernel.

Proof: By Theorem 1 for $\mathcal{P}=\mathcal{P}^{+}$we obtain that the number of conjugacy classes of orientable subgroups of index $2 n$ in the group $\Gamma$ is given by the formula

$$
c_{\Gamma}^{+}(2 n)=\frac{1}{2 n} \sum_{\substack{\ell \mid 2 n \\ \ell m=2 n}}\left(\sum_{K^{+}<_{m} \Gamma}\left|\operatorname{Epi}\left(K^{+}, \mathbb{Z}_{\ell}\right)\right|+\sum_{K^{-}<_{m} \Gamma}\left|\operatorname{Epi}^{+}\left(K^{-}, \mathbb{Z}_{\ell}\right)\right|\right),
$$

where the sums $\sum_{K^{+}<_{m} \Gamma}$ and $\sum_{K^{-}<_{m} \Gamma}$ are taken over all orientable and non-orientable subgroups of index $m$ in the group $\Gamma$, respectively and $\mathrm{Epi}^{+}\left(K^{-}, \mathbb{Z}_{\ell}\right)$ is the set of epimorphisms of the group $K^{-}$ onto a cyclic group $\mathbb{Z}_{\ell}$ of order $\ell$ with orientable kernel. We note that all orientable subgroups in $\Gamma$ are of even index. Hence, the condition $\sum_{K^{+}{ }_{{ }_{m} \Gamma} \Gamma}$ can be rewritten in the form $\sum_{K^{+}{ }_{{ }_{m} \Gamma^{+}}}$, where $m=2 \bar{m}$ and $\ell \bar{m}=n$. By the same reason any orientable kernel of the epimorphism of $K^{-}$onto $\mathbb{Z}_{\ell}$ has an even index in $K^{-}$. Hence, $\ell$ is even and $\mathrm{Epi}^{+}\left(K^{-}, \mathbb{Z}_{\ell}\right)$ can be represented in the form Epi ${ }^{+}\left(K^{-}, \mathbb{Z}_{2} \bar{\ell}\right)$, where $\bar{\ell} m=n$. After these remarks, by replacing $\bar{\ell}$ to $\ell$ an $\bar{m}$ to $m$ we obtain the result.

To make the above solution more explicit we have to calculate the numbers of epimorphisms $\left|\operatorname{Epi}\left(K^{+}, \mathbb{Z}_{\ell}\right)\right|$ and $\left|\mathrm{Epi}^{+}\left(K^{-}, \mathbb{Z}_{2 \ell}\right)\right|$. This will be done in the next section.

\section{Counting orientation preserving homomorphisms}

In this section we describe how to calculate orientation preserving epimorphisms of a given finitely generated group onto the cyclic group. We will follow the ideas given in papers (9), (13) and (14).

Let $\Gamma=(\Gamma, \omega)$ be a finitely generated group with sign structure. We represent the oriented homology group $\left(H_{1}(\Gamma), \bar{\omega}\right)$ of the group $\Gamma$ in the form

$$
H_{1}(\Gamma)=\mathbb{Z}_{k_{1}}^{\varepsilon_{1}} \oplus \mathbb{Z}_{k_{2}}^{\varepsilon_{2}} \oplus \cdots \oplus \mathbb{Z}_{k_{n}}^{\varepsilon_{n}}
$$

where $k_{j} \in\{2,3, \ldots, \infty\}$ and the notation $\mathbb{Z}_{k}^{\varepsilon}$ means that the corresponding cyclic group $\mathbb{Z}_{k}=\langle x| x^{k}=$ $1\rangle$ is generated by an element $x$ with $\bar{\omega}(x)=\varepsilon$ for some $\varepsilon \in\{-1,1\}$.

We accept the following conventions $\mathbb{Z}_{k}^{-1}=\mathbb{Z}_{k}^{-}, \quad \mathbb{Z}_{k}^{+1}=\mathbb{Z}_{k}^{+}, \mathbb{Z}_{\infty}=\mathbb{Z},(-1)^{\infty}=1,(k, \ell)=$ $\operatorname{gcd}(k, \ell)$ and $(\infty, \ell)=\ell$ for any positive integer $\ell$. 
EXAMPLE 4 Let $F_{r}=\left\langle x_{1}, x_{2}, \ldots, x_{r}\right\rangle$ be the free group of rank $r$ with a sign structure given by $\omega\left(x_{i}\right)=$ $-1, i=1,2, \ldots r$. Then $H_{1}\left(F_{r}\right)=\left(\mathbb{Z}^{-}\right)^{r}$.

EXAMPLE 5 Let $\Lambda_{p}=\left\langle x_{1}, x_{2}, \ldots, x_{p}: x_{1}^{2} x_{2}^{2} \cdots x_{p}^{2}=1\right\rangle$ be the fundamental group of a closed nonorientable surface of genus $p$ with sign structure given by $\omega\left(x_{i}\right)=-1, i=1, \ldots, p$. Then $H_{1}\left(\Lambda_{p}\right)=$ $\left(\mathbb{Z}^{-}\right)^{(p-1)} \oplus \mathbb{Z}_{2}^{(-1)^{p}}$

The main result is given by the following theorem obtained in (14).

Theorem 4 Let $\Gamma$ be a finitely generated group with non-trivial sign structure and

$$
H_{1}(\Gamma)=\mathbb{Z}_{k_{1}}^{\varepsilon_{1}} \oplus \mathbb{Z}_{k_{2}}^{\varepsilon_{2}} \oplus \cdots \oplus \mathbb{Z}_{k_{n}}^{\varepsilon_{n}}
$$

is the oriented homology group of $\Gamma$. Then $\left|\mathrm{Epi}^{+}\left(\Gamma, \mathbb{Z}_{\ell}\right)\right|=0$ if $\ell$ is odd and

$$
\left|\mathrm{Epi}^{+}\left(\Gamma, \mathbb{Z}_{2 \ell}\right)\right|=\prod_{j=1}^{n} \frac{\frac{k_{j}}{\left(k_{j}, \ell\right)}}{2} \sum_{\substack{\frac{\ell}{m}: \text { odd } \\ \text { in }}} \mu\left(\frac{\ell}{m}\right)\left(k_{1}, m\right)\left(k_{2}, m\right) \ldots\left(k_{n}, m\right) .
$$

We note that a similar formula for the number $\left|\operatorname{Epi}\left(\Gamma, \mathbb{Z}_{\ell}\right)\right|$ of all epimorphisms $\Gamma$ onto $Z_{\ell}$ was obtained in (21). Then we also have

$$
\left|\operatorname{Epi}^{-}\left(\Gamma, \mathbb{Z}_{\ell}\right)\right|=\left|\operatorname{Epi}\left(\Gamma, \mathbb{Z}_{\ell}\right)\right|-\left|\operatorname{Epi}^{+}\left(\Gamma, \mathbb{Z}_{\ell}\right)\right|
$$

\section{Application to surface coverings}

In this section we employ the above general theorems to count orientable coverings over bordered and and non-orientable surfaces. By straightforward calculations from Theorems 3 and 4 we obtain the following results.

Theorem 5 Let $\mathcal{B}$ be a bordered non-oriented surface with the fundamental group $\pi_{1}(\mathcal{B})=F_{r}$. Then the number of orientable $2 n$-fold coverings of $\mathcal{B}$ is given by the formula

$$
c_{F_{r}}^{+}(2 n)=\frac{1}{2 n} \sum_{\substack{\ell \mid n \\ \ell m=n}}\left(\varphi_{2 m(r-1)+1}(\ell) s_{F_{2 r-1}}(m)+\varphi_{m(r-1)+1}^{o d d}(\ell) s_{F_{r}}^{-}(m)\right),
$$

where $\varphi_{r}(\ell)$ and $\varphi_{r}^{\text {odd }}(\ell)$ are the Jordan functions, $s_{F_{r}}(m)$ is the numbers s of subgroups of index $m$, and $s_{F_{r}}^{-}(m)$ is the numbers of non-orientable subgroups of index $m$ in the group $F_{r}$.

We note that $c_{F_{r}}^{+}(2 n)$ coincides with the number of balanced $2 n$-fold coverings of a unbalanced graph $B_{r}$ obtained in (2). 
Theorem 6 Let $\mathcal{N}_{p}$ be a closed non-oriented surface of genus $p$ with the fundamental group $\pi_{1}\left(\mathcal{N}_{p}\right)=$ $\Lambda_{p}$. Then the number of orientable $2 n$-fold coverings of $\mathcal{N}_{p}$ is given by the formula

$$
c_{\Lambda_{r}}^{+}(2 n)=\frac{1}{2 n} \sum_{\substack{\ell \mid n \\ \ell m=n}}\left(\varphi_{2 s+1}(\ell) s_{\Lambda_{2(p-1)}}(m)+\frac{1+(-1)^{(s-1)(\ell-1)}}{2}(2, \ell) \varphi_{s}^{\text {odd }}(\ell) s_{\Lambda_{p}}^{-}(m)\right)
$$

where $s=m(p-2)+1, \varphi_{s}(\ell)$ and $\varphi_{s}^{o d d}(\ell)$ are the Jordan functions, $s_{\Lambda_{p}}(m)$ is the number of subgroups of index $m$, and $s_{\Lambda_{p}}^{-}(m)$ is the number of non-orientable subgroups of index $m$ in the group $\Lambda_{p}$.

The last theorem was obtained earlier in (2) by a combinatorial argument.

Let $\mathcal{K}$ be a Klein bottle, that is a closed non-oriented surface of genus 2 . It was shown in (20) and (10) that the number $c_{\mathcal{K}}^{-}(n)$ of $n$-fold coverings of $\mathcal{K}$ is expressed in terms of classical number-theoretical functions. The number $c_{\mathcal{K}}^{-}(n)$ of non-orientable $n$-fold coverings of $\mathcal{K}$ was calculated in (17) by a complicated method based on analysis of the subgroup structure in the fundamental group of $\mathcal{K}$.

Now, as a corollary of Theorem 2 we obtain a simple formula for $c_{\mathcal{K}}^{-}(n)$.

Recall that any positive integer $n$ can be uniquely represented in the form $n=2^{s} \cdot n^{-}$, where $s \geq 0$ and $n^{-}$is an odd number. We call $n^{+}=2^{s}$ and $n^{-}$by even and odd part of $n$, respectively.

Theorem 7 Let $\mathcal{K}$ be a Klein bottle. Then the number of non-orientable $n$-fold coverings of $\mathcal{K}$ is given by the formula

$$
c_{\mathcal{K}}^{-}(n)=(2, n) d\left(n^{-}\right),
$$

where $n^{-}$is the odd part of $n$ and $d(n)$ is the number of positive divisors of $n$.

\section{Reflexible coverings and chiral pairs}

Let $\mathcal{M}$ be a non-orientable manifold or orbifold. An orientable coverings $\pi: \mathcal{U}^{+} \rightarrow \mathcal{M}$ is called reflexible if there exists an orientation reversing homeomorphism $h: \mathcal{U}^{+} \rightarrow \mathcal{U}^{+}$such that $\pi \circ h=\pi$ and irreflexible (or chiral) otherwise. All irreflexible coverings are divided into chiral pairs of twins. Two twins are non-equivalent as coverings over $\mathcal{M}^{+}$, but have to be equivalent as coverings over $\mathcal{M}$.

In particular, any regular covering $\pi$ is reflexible. The multiplicity of any finite sheeted reflexible covering is an even number.

The following theorem is a consequence of Theorem 1 and Theorem 2:

Theorem 8 Let $\mathcal{M}$ be a connected non-orientable manifold with a finitely generated fundamental group $\Gamma$. Then the number of $2 n$-fold reflexible coverings of $\mathcal{M}$ is given by the formula

$$
a_{\Gamma}(n)=\frac{1}{n} \sum_{\substack{\ell \mid n \\ \ell m=n}} \sum_{K^{-}<_{m} \Gamma}\left|\operatorname{Epi}^{+}\left(K^{-}, \mathbb{Z}_{2 \ell}\right)\right|
$$

where the sum $\sum_{K^{-}}<_{m} \Gamma$ is taken over all non-orientable subgroups of index $m$ in the group $\Gamma$ and $\operatorname{Epi}^{+}\left(K, \mathbb{Z}_{\ell}\right)$ is the set of epimorphisms of the group $K$ onto a cyclic group $\mathbb{Z}_{\ell}$ of order $\ell$ with orientable kernel. 
Proof: Let $\Gamma^{+}$be the positive subgroup of $\Gamma$ and $\Gamma=\Gamma^{+}+\sigma \Gamma^{+}$is its coset decomposition. Let $K$ be a subgroup of $\Gamma^{+}$. Denote by $[K]_{\Gamma^{+}}$and $[K]_{\Gamma}$ the conjugacy class of $K$ in $\Gamma^{+}$and $\Gamma$, respectively.

There are two kinds of subgroups $K$ in $\Gamma^{+}$. Either reflexible with the property $[K]_{\Gamma^{+}}=\left[K^{\sigma}\right]_{\Gamma^{+}}=$ $[K]_{\Gamma}$ or twin with $[K]_{\Gamma^{+}} \neq\left[K^{\sigma}\right]_{\Gamma^{+}}$and $[K]_{\Gamma}=[K]_{\Gamma^{+}} \cup\left[K^{\sigma}\right]_{\Gamma^{+}}$. By definition, the set of all orientable subgroups is the disjoint union of reflexible and twin subgroups. Denote by $a_{\Gamma}(n)$ and $t_{\Gamma}(n)$ the numbers of conjugacy classes of reflexible and twin subgroups of index $2 n$ in the group $\Gamma$, respectively. Now we calculate the numbers of orientable subgroups of index $2 n$ up to conjugacy in $\Gamma$ and $\Gamma^{+}$. We get

$$
\begin{aligned}
& c_{\Gamma}^{+}(2 n)=a_{\Gamma}(n)+t_{\Gamma}(n) \\
& c_{\Gamma^{+}}(n)=a_{\Gamma}(n)+2 t_{\Gamma}(n) .
\end{aligned}
$$

From Theorem 3 we have

$$
c_{\Gamma}^{+}(2 n)=\frac{1}{2}\left(c_{\Gamma^{+}}(n)+I(n)\right)
$$

where

$$
I(n)=\frac{1}{n} \sum_{\substack{\ell \mid n \\ \ell m=n}} \sum_{K^{-}<_{m} \Gamma}\left|\mathrm{Epi}^{+}\left(K^{-}, \mathbb{Z}_{2 \ell}\right)\right| .
$$

Hence,

$$
a_{\Gamma}(n)=2 c_{\Gamma}^{+}(2 n)-c_{\Gamma^{+}}(n)=\left(c_{\Gamma^{+}}(n)+I(n)\right)-c_{\Gamma^{+}}(n)=I(n) .
$$

Also, since $t_{\Gamma}(n)=\frac{1}{2}\left(c_{\Gamma^{+}}(n)-a_{\Gamma}(n)\right)$ we obtain the following proposition.

Proposition 2 Let $\mathcal{M}^{+}$be the orientable double of a non-orientable manifold $\mathcal{M}$. Then the number of chiral pairs of $n$-fold coverings of $\mathcal{M}^{+}$is given by the formula

$$
t_{\Gamma}(n)=\frac{c_{\Gamma^{+}}(n)-a_{\Gamma}(n)}{2}
$$

where $\Gamma^{+}$is the fundamental group of $\mathcal{M}^{+}$and $c_{\Gamma^{+}}(n)$ and $a_{\Gamma}(n)$ are determined by Theorems 1 and 8 , respectively.

We illustrate results of the last section by the following example. Let $\mathcal{M}=\mathcal{K}$ be a Klein bottle with fundamental group

$$
\Gamma=\pi_{1}(\mathcal{K})=\left\langle x, y \mid x y x y^{-1}=1\right\rangle .
$$

The orientable double of $\mathcal{K}$ is a torus $\mathcal{T}$ with fundamental group

$$
\Gamma^{+}=\pi_{1}(\mathcal{T})=\langle a, b \mid[a, b]=1\rangle,
$$

where $a=x$ and $b=y^{2}$. We note that $\Gamma=\Gamma^{+}+\Gamma^{+} y, y a y^{-1}=a^{-1}$, and $y b y^{-1}=b$. 


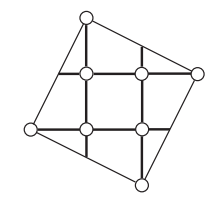

$<a^{-2} b, a b^{2}>$

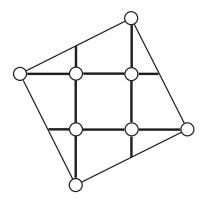

(i)

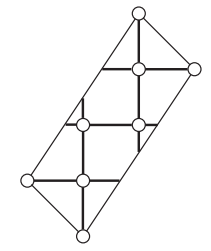

$<a^{-1} b, a^{2} b^{3}>$

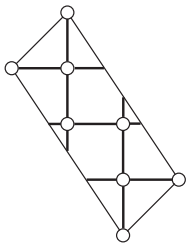

$<a b, a^{-2} b^{3}>$

(ii)

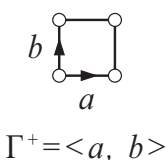

(iii)

Fig. 1

\section{The chiral pairs of coverings}

Set $n=5$. Then, by direct calculation from Theorems 1 and 6 we obtain the following equations $c_{\Gamma}^{+}(10)=a_{\Gamma}(5)+t_{\Gamma}(5)=4$ and $c_{\Gamma^{+}}(5)=a_{\Gamma}(5)+2 t_{\Gamma}(5)=6$. Hence, $a_{\Gamma}(5)=t_{\Gamma}(5)=2$.

That is the group $\Gamma^{+}$has six (conjugacy classes of) subgroups producing coverings. Two of them, $\left\langle a^{5}, b\right\rangle$ and $\left\langle a, b^{5}\right\rangle$ are reflexible and other four are divided into chiral pairs. They are $\left\langle a^{2} b, a^{-1} b^{2}\right\rangle,\left\langle a^{-2} b, a b^{2}\right\rangle$ and $\left\langle a b, a^{-2} b^{3}\right\rangle,\left\langle a^{-1} b, a^{2} b^{3}\right\rangle$, respectively. (See Fig. 1(i), (ii)).

The twin subgroups in chiral pairs are not conjugate in $\Gamma^{+}$but are conjugate by element $y$ in the group $\Gamma$.

\section{Numerical tables}

\begin{tabular}{|l|l|l|l|l|l|}
\hline$n$ & Total number & Non-orientable & Orientable & Reflexible & Chiral pairs \\
\hline 01 & 1 & 1 & 0 & 0 & 0 \\
\hline 02 & 3 & 2 & 1 & 1 & 0 \\
\hline 03 & 2 & 2 & 0 & 0 & 0 \\
\hline 04 & 5 & 2 & 3 & 3 & 0 \\
\hline 05 & 2 & 2 & 0 & 0 & 0 \\
\hline 06 & 7 & 4 & 3 & 2 & 1 \\
\hline 07 & 2 & 2 & 0 & 0 & 0 \\
\hline 08 & 8 & 2 & 6 & 5 & 1 \\
\hline 09 & 3 & 3 & 0 & 0 & 0 \\
\hline 10 & 8 & 4 & 4 & 2 & 2 \\
\hline 11 & 2 & 2 & 0 & 0 & 0 \\
\hline 12 & 13 & 4 & 9 & 6 & 3 \\
\hline
\end{tabular}

Table 1. The number of $n$-fold coverings of genus 2 non-orientable surface (Klein bottle) 


\begin{tabular}{|l|l|l|l|}
\hline$n$ & Total number & Non-orientable & Orientable \\
\hline 01 & 1 & 1 & 0 \\
\hline 02 & 7 & 6 & 1 \\
\hline 03 & 14 & 14 & 0 \\
\hline 04 & 89 & 80 & 9 \\
\hline 05 & 264 & 264 & 0 \\
\hline 06 & 1987 & 1930 & 57 \\
\hline 07 & 11086 & 11086 & 0 \\
\hline 08 & 93086 & 92240 & 846 \\
\hline 09 & 779579 & 779579 & 0 \\
\hline 10 & 7753717 & 7738414 & 15303 \\
\hline 11 & 82768656 & 82768656 & 0 \\
\hline 12 & 990033082 & 989476104 & 556978 \\
\hline
\end{tabular}

Table 2. The number of $n$-fold coverings of genus 3 non-orientable surface

\begin{tabular}{|l|l|l|l|}
\hline$n$ & Total number & Non-orientable & Orientable \\
\hline 01 & 1 & 1 & 0 \\
\hline 02 & 15 & 14 & 1 \\
\hline 03 & 82 & 82 & 0 \\
\hline 04 & 1583 & 1544 & 39 \\
\hline 05 & 30242 & 30242 & 0 \\
\hline 06 & 1110935 & 1109452 & 1483 \\
\hline 07 & 51724322 & 51724322 & 0 \\
\hline 08 & 3296935883 & 3296581880 & 354003 \\
\hline 09 & 264893065443 & 264893065443 & 0 \\
\hline 10 & 26438156395760 & 26437948184476 & 208211284 \\
\hline 11 & 3193922150539634 & 3193922150539634 & 0 \\
\hline 12 & 459644128922143309 & 459643859463061456 & 269459081853 \\
\hline
\end{tabular}

Table 3. The number of $n$-fold coverings of genus 4 non-orientable surface

\section{Acknowledgements}

The last two authors are grateful to the Combinatorial and Computational Mathematics Center at Pohang University of Science and Technology for hospitality during preparation of this paper. We are thankful to referees for helpful remarks and suggestions. 


\section{References}

[1] A. Breda, A. D. Mednykh, R. Nedela, Counting unrooted unsensed maps on closed orientable surface, to appear.

[2] I. P. Goulden, J. H. Kwak, J. Lee, Enumerating branched orientable surface coverings over a nonorientable surface, Discrete Math. 303 (2005), 42-55.

[3] P. Hall, The Euclidean functions of a group, Quart. J. Math. Oxford 7 (1936), 134-151.

[4] M. Hall, Jr., Subgroups of finite index in free groups, Canadian J. Math. 1 (1949), 187-190.

[5] A. Hatcher, Algebraic Topology, Cambridge University Press, Cambridge, 2002.

[6] M. Hofmeister, A note on counting connected graph covering projections, SIAM J. Discrete Math. 11 (1998), 286-292.

[7] A. Hurwitz, Über Riemann'sche Flächen mit gegebenen Verzweigungspunkten, Math. Ann. 39 (1891), 1-61.

[8] A. Hurwitz, Über die Anzahl der Riemann'sche Flächen mit gegebenen Verzweigungspunkten, Math. Ann. 55 (1902), 53-66.

[9] G. A. Jones, Enumeration of homomorphisms and surface-coverings, Quart. J. Math. Oxford (2) 46 (1995), 485-507.

[10] G. A. Jones and, V. A. Liskovets, Enumarating subgroups of finite index, in: Proceedings of $\mathrm{Com}^{2} \mathrm{MaC}$ Mini-Workshop on Hurwitz Theory and Ramifications (January 13-18, 2003), editors J. H. Kwak and A. D. Mednykh, Combinatorial and Computational Mathemetics Center, Pohang University of Science and Technology, Pohang, 2003.

[11] J. H. Kwak and J. Lee, Isomorphism classes of graph bundles, Canad. J. Math. XLII (1990), 747761.

[12] J. H. Kwak and J. Lee, Enumeration of connected graph coverings, J. Graph Theory 23 (1996), $105-109$.

[13] J. H. Kwak, J. Lee and Y. Shin, Balanced coverings of a signed graph and some regular branched orientable surface coverings over a non-orientable surface, Discrete Math. 275 (2004), 177-193.

[14] J. H. Kwak, R. Nedela, A. D. Mednykh, On the number of orientable coverings over non-orientable manifold, to appear.

[15] V. Liskovets, On the enumeration of subgroups of a free group, Dokl. Akad. Nauk BSSR. 15 (1971), 6-9.

[16] V. Liskovets, Reductive enumeration under mutually orthogonal group actions, Acta Applicandae Mathematicae 52 (1998), 91-120. 
[17] V. Liskovets and A. Mednykh, The number of non-equivalent unbranched $n$-fold coverings of the Klein bottle, preprint (2002), com2mac.postech.ac.kr/papers/2002/02-06.pdf.

[18] V. Liskovets and A. Mednykh, On the number of disconnected coverings over manifold, preprint (2006), com2mac.postech.ac.kr/papers/2007/03-20.pdf.

[19] A. D. Mednykh, Hurwitz problem on the number of nonequivalent coverings of a compact Riemann surface, Siber. Math. J. 23 (1982), 415-420.

[20] A. D. Mednykh, On the number of subgroups in the fundamental group of a closed surface, Comm. Algebra 16 (1988), 2137-2148.

[21] A. D. Mednykh, A new method for counting coverings over manifold with finitely generated group, Doklady Mathematics 74(1) (2006), 498-502.

[22] A. D. Mednykh, R. Nedela, Counting unrooted hypermaps on closed orientable surface, Proceedings of the 18th International Conference on Formal Power Series and Algebraic Combinatorics, June 19-23, 2006, San Diego, California, 2006, 1-19.

[23] A. D. Mednykh and G. G. Pozdnyakova, Number of nonequivalent coverings over a non-orientable compact surface, Siber. Math. J. 27 (1986), 99-106.

[24] J. Šráň and M. S̆koviera, Groups with sign structure and their antiautomorphisms, Discrete Math. 108 (1992), 189-202. 